\section{Assistência pré-natal nos serviços públicos e privados de saúde: estudo transversal de base populacional em Rio Grande, Rio Grande do Sul, Brasil}

\author{
Prenatal care in public and private health services: \\ a population-based survey in Rio Grande, Rio \\ Grande do Sul State, Brazil
}

\author{
${ }^{1}$ Divisão de População \& \\ Saúde, Universidade Federal \\ do Rio Grande, Rio Grande, \\ Brasil. \\ 2 Programa de Pós-graduação \\ em Epidemiologia, \\ Universidade Federal de \\ Pelotas, Pelotas, Brasil. \\ Correspondência \\ J. A. Cesar \\ Divisão de População \& \\ Saúde, Universidade Federal \\ do Rio Grande. \\ Rua General Osório s/n, \\ Rio Grande, $R S$ \\ 96201-900, Brasil. \\ juraci.cesar@gmail.com
}

\begin{abstract}
This study aimed to evaluate public and private prenatal care for women in Rio Grande, Rio Grande do Sul State, Brazil. Women who gave birth at the two local maternity hospitals from January 1 to December 31, 2010, answered a standardized questionnaire. The interview sites in the public sector were primary health care units with and without the Family Health Strategy (FHS) and outpatient clinics; the private sector included clinics operated by health plans and private physicians' offices. The chi-square test was used to compare proportions. The response rate was $97.2 \%$ (2,395 out of 2,464). Among the 23 target variables and indicators, seven showed a clear advantage for mothers who had received prenatal care under the FHS and six for health plan clinics and private offices. Four variables showed virtually universal coverage at all five study sites. Prenatal care showed better coverage for pregnant women treated in the private sector. Pregnant women treated under the FHS showed similar coverage to that in the private sector.
\end{abstract}

Prenatal Care; Health Services Evaluation; Health Services Coverage
Juraci A. Cesar 1,2

Andréa T. Sutil 1

Gabriela B. dos Santos 1

Carolina F. Cunha 1

Raúl A. Mendoza-Sassi 1

\section{Introdução}

A oferta de pré-natal adequado reduz a morbimortalidade materno-infantil 1. O número de consultas de pré-natal está diretamente associado ao melhor resultado gestacional, e isto se expressa em termos de melhor crescimento intrauterino, maior peso ao nascer, menor ocorrência de prematuridade, de mortalidade neonatal e de adoecimento e morte entre mães 2,3,4.

Nas últimas duas décadas, houve, por parte do setor público brasileiro, importante investimento na assistência básica em saúde. Em 1994, o Governo Federal lançou o Programa Saúde da Família, atual Estratégia Saúde da Família (ESF), com o objetivo de reorientar o modelo assistencial por meio da oferta de cuidados nas unidades básicas de saúde (UBS). Atualmente, são cerca de 30 mil equipes multiprofissionais presentes em aproximadamente 5,2 mil municípios brasileiros. Isso levou a um substancial aumento da cobertura da assistência pré-natal, sobretudo nas áreas mais carentes 5 .

No ano 2000, o Ministério da Saúde instituiu o Programa de Humanização no Pré-natal e Nascimento (PHPN). Um dos principais objetivos dessa iniciativa foi assegurar acesso, melhorar a cobertura e a qualidade do pré-natal oferecido, além de preparar as unidades de saúde para receber de forma acolhedora a gestante, seus familiares e o recém-nascido 4. 
A assistência pré-natal no Brasil vem melhorando substancialmente ${ }^{6}$. Vários procedimentos clínicos e exames laboratoriais aproximam-se da universalização. Isso tem sido observado em diferentes localidades e em diferentes regiões do país 7,8,9,10. Apesar disso, não são poucas as publicações a assegurar que o pré-natal realizado no setor privado é melhor que aquele oferecido no setor público 3,11,12. Essa melhoria implica maior cobertura para os diversos indicadores da assistência pré-natal entre gestantes atendidas no setor público em relação àquelas atendidas no setor privado.

Este artigo teve por objetivo estimar a cobertura para diversos exames laboratoriais, procedimentos clínicos e orientações recebidas durante o pré-natal, entre gestantes atendidas em UBS com e sem ESF, ambulatórios públicos e clínicas de convênio, e consultórios particulares em Rio Grande, município de porte médio localizado no extremo sul do Brasil.

\section{Metodologia}

O Município de Rio Grande possui cerca de 200 mil habitantes, é litorâneo, está localizado a $300 \mathrm{~km}$ de Porto Alegre (capital do Estado do Rio Grande do Sul) e a $250 \mathrm{~km}$ da divisa com o Uruguai, e a atividade portuária é, atualmente, sua principal fonte de renda. Em 2010, possuía 197 mil habitantes, taxa de alfabetização de $95,4 \%$, expectativa de vida ao nascer de 69 anos, coeficiente de mortalidade infantil de 11,4/1.000 e, neste ano, não houve um único óbito materno no município. O produto interno bruto per $\mathrm{ca}$ pita era de $\mathrm{R} \$ 32$ mil e, dentre os 496 municípios gaúchos, ocupava a 10a posição no quesito índice de desenvolvimento socioeconômico e a 435a em termos de indicadores de saúde (Fundação de Economia e Estatística Siegfried Emanuel Heuser. Resumo estatístico RS. http://www.fee.tche. $\mathrm{br} /$ sitefee/pt/content/resumo/pg_municipios_ detalhe.php?municipio=Rio+Grande, acessado em 22/Jun/2012).

Os dados apresentados neste trabalho fazem parte do estudo Perinatal_2010, conduzido neste município. Este estudo incluiu todos os nascimentos com peso igual ou superior a 500 gramas ou pelo menos 20 semanas de idade gestacional, ocorridos nas maternidades da Santa Casa de Misericórdia e no Hospital Universitário da Universidade Federal do Rio Grande (HU-FURG), entre 1o de janeiro e 31 de dezembro de 2010. Além disso, suas mães deveriam residir em área rural ou urbana do município. O delineamento utilizado foi do tipo transversal (seccional ou de prevalência) com as mães sen- do entrevistadas na maternidade por ocasião do parto ${ }^{13}$.

Todas as informações foram coletadas por meio de questionário único pré-codificado, com a quase totalidade das perguntas fechadas e aplicado à mãe em até 24 horas após o parto. Esse questionário buscou informações sobre o local de residência da família, características demográficas, ocupação, história reprodutiva e hábitos de vida das mães; nível socioeconômico, posse de eletrodomésticos e condições de habitação e saneamento de suas famílias, além de características do trabalho do pai; assistência recebida durante a gestação e o parto, acesso e utilização de serviços preventivos e curativos em saúde, e imunização antitetânica. A idade gestacional do recém-nascido foi obtida a partir da data da última menstruação (DUM) anotada no cartão da gestante, ou referida pela mãe e por ultrassonografia realizada entre a 6 a e a $20 \underline{a}$ semanas de gestação.

Em cada um dos locais de realização do prénatal perguntou-se à mãe o nome do médico que a atendeu na maioria das consultas. No caso das UBS, com base em lista fornecida pela Secretaria Municipal de Saúde verificava-se se aquele profissional pertencia de fato a ESF. Em caso de dúvida, contactava-se a coordenação do programa. Se a gestante realizou consulta de pré-natal em mais de um local, foi computado como local de realização aquele em que ela realizou o maior número de consultas. No caso de ter feito o mesmo número de consultas em diferentes locais, definiu-se como local de realização aquele em que ela concluiu o maior número de consultas no primeiro trimestre de gravidez, depois no segundo e, por último, no terceiro.

A adequação do pré-natal foi avaliada segundo critérios propostos por Takeda 14 e Silveira et al. 15 . Takeda 14 combina o número total de consultas com o momento de ingresso ao pré-natal e três categorias são estabelecidas: "adequado", para seis ou mais consultas de pré-natal antes de cinco meses de gestação; "inadequado", menos de três consultas e se estas ocorrem após o sétimo mês de gestação; e "intermediário", que engloba as demais situações. Silveira et al. 15 consideram o pré-natal como "adequado" se forem realizadas seis ou mais consultas e se o início aconteceu antes do quinto mês de gestação, mais a realização de pelo menos dois exames qualitativos de urina, hemoglobina e VDRL; "inadequado" para três consultas com início após o sétimo mês de gestação, sem a realização de qualquer um dos exames citados; e "intermediário" para as demais situações. Nos dois critérios, dados não lembrados pelas gestantes foram classificados na categoria "ignorado". 
Três entrevistadoras graduadas na área de ciências humanas foram contratadas para realizar essas entrevistas. O treinamento das entrevistadoras consistiu de leitura do questionário e do manual de instruções e de simulação de entrevistas. O estudo piloto foi realizado na primeira quinzena de dezembro de 2009 nos mesmos locais onde foi, posteriormente, realizada a coleta de dados. Essas entrevistadoras permaneceram por ocasião da coleta de dados em regime de rodízio mensal nas duas maternidades. Mães residentes no Município de Rio Grande foram convidadas a participar do estudo, ocasião em que assinavam o Termo de Consentimento autorizando a realização da entrevista. Uma cópia desse termo ficava de posse de cada uma delas.

Os questionários aplicados foram codificados pelas próprias entrevistadoras ao final de cada dia de trabalho e entregues na sede do projeto, onde todos foram revisados. Em seguida, utilizandose o programa Epidata 3.1 (Epidata Association, Odense, Dinamarca), esses questionários foram digitados e corrigidos para valores inconsistentes. A análise dos dados, que constou da comparação de proporções pelo teste qui-quadrado, foi realizada utilizando-se o Stata 11.2 (Stata Corp., College Station, Estados Unidos).

$\mathrm{O}$ controle de qualidade constou de repetição de cerca de $5 \%$ das entrevistas, sendo estas realizadas, na maioria das vezes, por telefone. $\mathrm{O}$ protocolo de pesquisa foi submetido e aprovado pelo Comitê de Ética em Pesquisa na Área da Saúde (CEPAS) da FURG. Além disso, garantiu-se à mãe confidencialidade dos dados coletados, a participação voluntária e a possibilidade de deixar o estudo a qualquer momento, sem necessidade de justificativa.

\section{Resultados}

Dentre as 2.464 gestantes residentes no Município de Rio Grande que tiveram filhos em 2010, $2.395(97,2 \%)$ foram incluídas neste trabalho. Para as outras 69,51 não foram encontradas e 18 recusaram-se a participar do estudo.

A Tabela 1 mostra que as parturientes que realizaram consultas de pré-natal no setor público eram, em média, três anos mais novas, apresentavam maior proporção de cor da pele parda e preta, viviam em menor proporção com companheiro, possuíam, em media, três anos a menos de escolaridade e cerca de metade da renda daquelas que realizaram o pré-natal no setor privado. Por fim, duas em cada três delas tiveram a primeira gravidez na adolescência, contra menos de um terço daquelas atendidas no setor privado.
A taxa de ocorrência de aborto foi a mesma nos dois grupos.

Na Tabela 2 é possível verificar vantagem sistemática para gestantes atendidas no setor privado para a realização de seis ou mais consultas de pré-natal, trimestre de início do pré-natal, efetivação de exames laboratoriais e clínicos, orientação sobre o uso de medicamentos e prática de exercício físico durante a gestação, realização do pré-natal com o mesmo médico e adequação do pré-natal. Gestantes atendidas no setor público mostraram vantagem significativa no que diz respeito à cobertura para vacinação antitetânica e suplementação com sulfato ferroso.

A Tabela 3 mostra a prevalência de realização de cada um dos procedimentos clínicos, exames laboratoriais e orientações recebidas durante as consultas de pré-natal de acordo com os cinco locais de consulta avaliados. Dentre os 23 indicadores considerados, sete deles apresentaram maior cobertura nos locais de atuação da ESF, seis em clínica de convênio e seis em consultório particular. Quatro dos indicadores avaliados (verificação do peso, da pressão arterial, altura uterina e data da última menstruação) mostraram cobertura praticamente universal em todos os locais de consulta estudados.

\section{Discussão}

Gestantes atendidas no setor público estiveram em desvantagem em relação àquelas atendidas no setor privado no que diz respeito a características demográficas, socioeconômicas, reprodutivas e de cobertura para a quase totalidade dos indicadores da assistência pré-natal avaliados. Em relação ao local de consulta, verificou-se que o maior número de indicadores com melhor cobertura ocorreu para gestantes atendidas na ESF, seguidas de perto por aquelas atendidas em clínicas de convênio e consultório particular. Quatro indicadores apresentaram cobertura praticamente universal em todos os locais estudados.

Ao interpretar esses dados há que se ter em mente que se trata de uma avaliação quantitativa, que considerou apenas a prevalência de realização de um determinado procedimento clínico, exame laboratorial ou orientação recebida durante a consulta de pré-natal. Não se teve acesso, por exemplo, ao tipo de orientação dada sobre amamentação ou uso de medicamentos. Também não se avaliou se um determinado procedimento clínico foi adequadamente realizado ou não. Além disso, cerca de $30 \%$ das mães atendidas no setor público e $25 \%$ no setor privado não realizaram pré-natal com o mesmo profissional, e o estudo não investigou estas mudanças 
Tabela 1

Características demográficas das puérperas e socioeconômicas de suas famílias incluídas neste estudo. Rio Grande, Rio Grande do Sul, Brasil, 2010.

\begin{tabular}{|c|c|c|c|}
\hline \multirow[t]{2}{*}{ Variável } & \multicolumn{2}{|c|}{ Setor (\%) } & \multirow[t]{2}{*}{ Total (\%) } \\
\hline & Público & Privado & \\
\hline \multicolumn{4}{|l|}{ Idade da mãe (anos) $[\mathrm{p}<0,001]$} \\
\hline $13-19$ & 25,2 & 9,1 & 18,4 \\
\hline $20-24$ & 28,7 & 23,4 & 26,4 \\
\hline $25-29$ & 23,4 & 29,7 & 26,0 \\
\hline 30 ou mais & 22,7 & 38,0 & 29,1 \\
\hline Média (desvio-padrão) & $24,7(6,4)$ & $27,7(6,0)$ & $25,9(6,4)$ \\
\hline \multicolumn{4}{|l|}{ Cor da pele autorreferida $[p<0,001]$} \\
\hline Branca & 62,3 & 79,0 & 69,4 \\
\hline Parda & 26,0 & 14,3 & 21,1 \\
\hline Preta & 11,7 & 5,5 & 9,5 \\
\hline Vivem junto com esposo/companheiro $[p<0,001]$ & 81,0 & 90,1 & $84,8 \%$ \\
\hline \multicolumn{4}{|l|}{ Escolaridade (anos completos de estudos) $[p<0,001]$} \\
\hline $0-8$ & 62,4 & 18,1 & 43,7 \\
\hline $9-11$ & 35,3 & 59,3 & 45,5 \\
\hline 12 ou mais & 2,3 & 22,5 & 10,8 \\
\hline Média (desvio-padrão) & $7,8(2,8)$ & $10,9(2,7)$ & $9,0(3,2)$ \\
\hline \multicolumn{4}{|l|}{ Renda familiar (quartis) $[p<0,001]$} \\
\hline Primeiro (menor) & 31,5 & 14,3 & 24,3 \\
\hline Segundo & 34,2 & 15,3 & 26,3 \\
\hline Terceiro & 23,0 & 24,8 & 23,7 \\
\hline Quarto (Maior) & 11,3 & 45,5 & 25,7 \\
\hline Média em Reais & $1,278,40$ & $2.425,78$ & $1.713,79$ \\
\hline \\
\hline \multicolumn{4}{|l|}{ Idade por ocasião da primeira gravidez (anos) $[p<0,001]$} \\
\hline $11-19$ & 66,5 & 29,5 & 50,9 \\
\hline $20-24$ & 23,2 & 29,4 & 25,8 \\
\hline $25-29$ & 7,2 & 26,2 & 15,2 \\
\hline 30 ou mais & 3,2 & 14,9 & 8,1 \\
\hline Média (desvio-padrão) & $19,1(4,2)$ & $23,4(5,6)$ & $20,8(5,3)$ \\
\hline \multicolumn{4}{|l|}{ Número de vezes que já engravidou $[p<0,001]$} \\
\hline 1 & 36,6 & 52,3 & 43,4 \\
\hline $2-3$ & 44,7 & 40,6 & 43,0 \\
\hline 4 ou mais & 18,7 & 6,8 & 13,7 \\
\hline Total [n (\%)] & $1.324(100,0)$ & $964(100,0)$ & $2.288(100,0)$ \\
\hline
\end{tabular}

dentro de um mesmo setor nem entre setores. Pelo menos localmente, não se tem conhecimento de qualquer ocorrência que justificasse mudança de gestantes, de forma sistemática, de um setor para outro. Ao que parece, isso ocorreu ao acaso. Por fim, as comparações aqui realizadas estão baseadas somente no relato das mães. Isso porque $49 \%$ não possuíam carteira da gestante no momento do parto e, além disso, para boa parte das que tinham a carteira, inúmeras informações não haviam sido preenchidas. Se esse possível viés de recordatório ocorreu, é razoável pensar que tenha afetado igualmente todas as gestantes.

Não encontramos nenhum artigo que tratasse de comparação semelhante a que apresentamos neste trabalho. Os que mais se aproximam dos resultados aqui mostrados comparam a cobertura de pré-natal entre os setores público e privado, mostrando nítida vantagem para as gestantes atendidas no setor privado 11,12 . As 
Tabela 2

Assistência pré-natal recebida de acordo com o tipo de serviço público ou privado utilizado. Rio Grande, Rio Grande do Sul, Brasil, 2010.

\begin{tabular}{|c|c|c|c|c|}
\hline \multirow[t]{2}{*}{ Variável } & \multicolumn{3}{|c|}{ Tipo de serviço utilizado pela puérpera (\%) } & \multirow[t]{2}{*}{ Valor de $\mathrm{p}$} \\
\hline & Público & Privado & Total & \\
\hline Realizaram seis ou mais consultas & 71,6 & 91,6 & 80,0 & $<0,001$ \\
\hline Iniciaram as consultas de pré-natal no primeiro trimestre de gravidez & 70,6 & 88,7 & 78,3 & $<0,001$ \\
\hline \multicolumn{5}{|l|}{ Exames laboratoriais realizados } \\
\hline Glicemia (2 ou mais) & 75,6 & 89,0 & 81,3 & $<0,001$ \\
\hline VDRL (2 ou mais) & 56,0 & 60,8 & 58,0 & 0,023 \\
\hline Exame comum de urina ( 2 ou mais) & 69,4 & 85,8 & 76,4 & $<0,001$ \\
\hline Anti-HIV (2 ou mais) & 62,8 & 65,3 & 63,8 & 0,216 \\
\hline Ultrassonografia (6a a 20a semana) & 56,7 & 71,8 & 63,3 & $<0,001$ \\
\hline Citopatológico de colo uterino & 62,9 & 72,9 & 67,1 & $<0,001$ \\
\hline \multicolumn{5}{|l|}{ Exames clínicos realizados } \\
\hline Pesagem & 99,3 & 99,4 & 99,3 & 0,867 \\
\hline Exame das mamas & 53,5 & 59,9 & 56,2 & 0,002 \\
\hline Verificação da pressão arterial & 98,1 & 99,7 & 98,8 & 0,001 \\
\hline Altura uterina & 99,3 & 98,6 & 99,0 & 0,106 \\
\hline Exame ginecológico & 57,8 & 76,3 & 65,6 & $<0,001$ \\
\hline \multicolumn{5}{|l|}{ Se durante a consulta o médico orientou sobre } \\
\hline Amamentação & 68,3 & 67,3 & 67,9 & 0,602 \\
\hline Uso de medicamentos & 85,8 & 91,6 & 88,2 & $<0,001$ \\
\hline Realização de exercícios físicos & 61,2 & 72,3 & 65,9 & $<0,001$ \\
\hline \multicolumn{5}{|l|}{ Se durante a consulta o médico perguntou sobre } \\
\hline Data da última menstruação & 99,5 & 99,3 & 99,4 & 0,55 \\
\hline Uso atual de medicamentos & 82,3 & 90,0 & 85,6 & $<0,001$ \\
\hline \multicolumn{5}{|l|}{ Se recebeu } \\
\hline Sulfato ferroso & 86,0 & 61,2 & 75,6 & $<0,001$ \\
\hline Vacina antitetânica & 81,5 & 74,3 & 78,5 & $<0,001$ \\
\hline Se fez todo o pré-natal com o mesmo médico & 72,1 & 88,3 & 78,9 & $<0,001$ \\
\hline \multicolumn{5}{|l|}{ Realizou pré-natal adequado conforme proposto por } \\
\hline Takeda 14 & 65,7 & 87,5 & 74,9 & $<0,001$ \\
\hline Silveira et al. 15 & 37,6 & 52,3 & 43,8 & $<0,001$ \\
\hline Total [n (\%)] & $1.324(100,0)$ & $964(100,0)$ & $2.228(100,0)$ & \\
\hline
\end{tabular}

Nota: Takeda 14 - combina o número total de consultas com o momento de ingresso ao pré-natal e três categorias são estabelecidas: "adequado", para seis ou mais consultas de pré-natal antes de cinco meses de gestação; "inadequado", menos de três consultas se estas ocorrem após o sétimo mês de gestação; e "intermediário", que engloba as demais situações; Silveira et al. 15 - este índice considera o pré-natal como "adequado" a realização de seis ou mais consultas com início antes do quinto mês de gestação e a realização de dois ou mais exames qualitativos de urina, hemoglobina e VDRL; "inadequado" para três consultas iniciadas após o sétimo mês de gestação sem a realização de qualquer um dos exames citados; e "intermediário" para as demais situações. Dados não lembrados pelas gestantes foram classificados na categoria "ignorado".

únicas exceções observadas nessas comparações dizem respeito à vacinação antitetânica e suplementação com sulfato ferroso que apresentam, em geral, maior cobertura no setor público. No caso da vacina antitetânica, sua oferta é quase uma exclusividade do sistema público de saúde, enquanto a suplementação com sulfato ferroso parece restringir-se às gestantes pobres.

Em relação às coberturas para os diversos indicadores da assistência pré-natal, é possível ga- rantir que, tanto no setor público quanto no setor privado, eles vêm melhorando sensivelmente nos últimos anos na localidade estudada. Entre 2007 e 2010, a proporção de mães riograndinas que completou seis ou mais consultas de pré-natal passou de $72 \%$ para $80 \%$, o inicio do pré-natal ainda no primeiro trimestre de gravidez passou de $74 \%$ para $78 \%$, enquanto a palpação das mamas e o exame ginecológico passaram de $45 \%$ para $56 \%$, e de $56 \%$ para $66 \%$, respectivamente. 
Tabela 3

Assistência pré-natal recebida de acordo com o tipo de médico que prestou atendimento. Rio Grande, Rio Grande do Sul, Brasil, 2010.

\begin{tabular}{|c|c|c|c|c|c|c|c|}
\hline \multirow[t]{2}{*}{ Variável } & \multicolumn{6}{|c|}{ Local de consulta (\%) } & \multirow{2}{*}{$\begin{array}{l}\text { Valor } \\
\text { de } p\end{array}$} \\
\hline & $\begin{array}{l}\text { UBS com } \\
\text { ESF }\end{array}$ & $\begin{array}{l}\text { UBS tradi- } \\
\text { cional }\end{array}$ & $\begin{array}{l}\text { Ambu- } \\
\text { latório }\end{array}$ & $\begin{array}{l}\text { Clínica de } \\
\text { convênio }\end{array}$ & $\begin{array}{c}\text { Consultório } \\
\text { particular }\end{array}$ & Total & \\
\hline Realizaram seis ou mais consultas & 81,9 & 64,2 & 67,4 & 92,0 & 90,8 & 80,0 & $<0,001$ \\
\hline \multicolumn{7}{|l|}{ 1으 trimestre } & $<0,001$ \\
\hline \multicolumn{8}{|l|}{ Exames laboratoriais realizados } \\
\hline Glicemia (2 ou mais) & 84,4 & 62,9 & 75,8 & 88,5 & 89,9 & 81,3 & $<0,001$ \\
\hline VDRL (2 ou mais) & 63,7 & 45,7 & 55,5 & 62,4 & 57,1 & 58,0 & 0,023 \\
\hline Exame comum de urina (2 ou mais) & 81,6 & 58,0 & 66,7 & 85,9 & 85,6 & 76,4 & $<0,001$ \\
\hline Anti-HIV (2 ou mais) & 70,2 & 52,1 & 62,8 & 66,6 & 62,5 & 63,8 & 0,216 \\
\hline Ultrassonografia (6a a 20ạ semana) & 54,8 & 50,9 & 61,3 & 72,7 & 70,0 & 63,3 & $<0,001$ \\
\hline Citopatológico de colo uterino & 54,9 & 31,7 & 40,7 & 30,8 & 28,8 & 37,8 & $<0,001$ \\
\hline \multicolumn{8}{|l|}{ Exames clínicos realizados } \\
\hline Pesagem & 99,6 & 99,0 & 99,3 & 99,4 & 99,3 & 99,3 & 0,867 \\
\hline Exame das mamas & 63,9 & 48,2 & 47,9 & 59,4 & 60,8 & 56,2 & 0,002 \\
\hline Verificação da pressão arterial & 99,6 & 99,0 & 96,4 & 99,9 & 99,0 & 98,8 & 0,1 \\
\hline Altura uterina & 99,8 & 99,0 & 99,1 & 98,6 & 98,7 & 99,0 & 0,106 \\
\hline Exame ginecológico & 62,4 & 49,5 & 58,7 & 77,3 & 74,2 & 65,6 & $<0,001$ \\
\hline \multicolumn{7}{|l|}{ Se durante a consulta o médico orientou } & \\
\hline Amamentação & 81,2 & 70,5 & 56,7 & 65,3 & 71,6 & 67,9 & 0,602 \\
\hline Uso de medicamentos & 90,3 & 86,0 & 82,0 & 91,2 & 92,5 & 88,2 & $<0,001$ \\
\hline Realização de exercícios físicos & 67,0 & 57,8 & 58,5 & 75,8 & 64,7 & 65,9 & $<0,001$ \\
\hline \multicolumn{7}{|l|}{ Se durante a consulta o médico perguntou } & \\
\hline Data da última menstruação & 99,6 & 99,0 & 99,6 & 99,1 & 99,7 & 99,4 & 0,55 \\
\hline Uso atual de medicamentos & 86,1 & 82,2 & 79,3 & 89,1 & 92,2 & 85,6 & $<0,001$ \\
\hline \multicolumn{8}{|l|}{ Se recebeu } \\
\hline Sulfato ferroso & 92,7 & 84,8 & 81,3 & 65,0 & 52,9 & 75,6 & $<0,001$ \\
\hline Vacina antitetânica & 88,1 & 82,0 & 75,9 & 77,3 & 67,7 & 78,5 & $<0,001$ \\
\hline \multicolumn{7}{|l|}{ médico } & $<0,001$ \\
\hline \multicolumn{8}{|l|}{ Realizou pré-natal adequado conforme } \\
\hline \multicolumn{8}{|l|}{ proposto por } \\
\hline Takeda 14 & 76,1 & 58,7 & 61,2 & 87,4 & 87,9 & 74,9 & $<0,001$ \\
\hline Silveira et al. 15 & 47,8 & 27,9 & 34,8 & 53,9 & 48,7 & 43,8 & $<0,001$ \\
\hline \multirow[t]{2}{*}{ Total $[n(\%)]$} & 452 & 315 & 557 & 658 & 306 & 2.288 & \\
\hline & $(100,0)$ & $(100,0)$ & $(100,0)$ & $(100,0)$ & $(100,00)$ & $(100,0)$ & \\
\hline
\end{tabular}

ESF: Estratégia Saúde da Família; UBS: unidade básica de saúde.

Nota: Takeda 14 - combina o número total de consultas com o momento de ingresso ao pré-natal e três categorias são estabelecidas: "adequado", para seis ou mais consultas de pré-natal antes de cinco meses de gestação; "inadequado", menos de três consultas se estas ocorrem após o sétimo mês de gestação; e "intermediário", que engloba as demais situações; Silveira et al. 15 - este índice considera o pré-natal como "adequado" a realização de seis ou mais consultas com início antes do quinto mês de gestação e a realização de dois ou mais exames qualitativos de urina, hemoglobina e VDRL; "inadequado" para três consultas iniciadas após o sétimo mês de gestação sem a realização de qualquer um dos exames citados; e "intermediário" para as demais situações. Dados não lembrados pelas gestantes foram classificados na categoria "ignorado". 
Quanto a exames laboratoriais, nesse mesmo período, HIV e VDRL passaram de $95 \%$ para $98 \%$, e de $94 \%$ para $97 \%$, respectivamente 16 . Em municípios pobres das regiões Norte e Nordeste do Brasil, entre 2002 e 2005, a realização de seis ou mais consultas de pré-natal aumentou de $42 \%$ para $52 \%$ e a imunização contra tétano neonatal de $54 \%$ para $77 \%$. Este mesmo estudo mostra que a avaliação da altura uterina passou de $54 \%$ para $77 \%$, enquanto a realização de teste para HIV e sífilis duplicou, de $8 \%$ para $16 \%$. A realização de ultrassonografia pélvica aumentou de $44 \%$ para $64 \% 6$. Em Juiz de Fora (Minas Gerais), entre 2002 e 2004, o número médio de consultas de pré-natal passou de 6,4 para 7,2, a idade gestacional na primeira consulta de 18 para 16 semanas, tipagem sanguínea de $78 \%$ para $84 \%$, medida de peso durante a consulta de $75 \%$ para $83 \%$, enquanto a verificação da altura uterina passou de $73 \%$ para $81 \%$. Isso fez com que a taxa de pré-natal adequado aumentasse de $37 \%$ para $65 \%$ 9. Em Pelotas, município vizinho com características semelhantes às de Rio Grande, entre 1982 e 2004, a proporção de mulheres que não realizaram uma única consulta de pré-natal caiu de $5 \%$ para $2 \%$, o número médio de consultas por gestante passou de 6,7 para 8,1 , enquanto que a proporção daquelas que iniciavam o pré-natal a partir do 5o mês de gestação caiu de $15 \%$ para $7 \% 17$. Todas as diferenças mencionadas foram estatisticamente significativas.

O que chama a atenção neste estudo é o fato de o pré-natal oferecido pela ESF, uma das modalidades do setor público, portanto do Sistema Único de Saúde (SUS), apresentar vários indicadores de cobertura melhores do que o seu correspondente no setor privado. Não encontramos isso descrito na literatura para um município de porte médio, portanto, com um número expressivo de gestantes, como é o caso de Rio Grande.
E não são indicadores menos relevantes (sorologia para sífilis, anti-HIV, citopatológico para colo uterino, suplementação com sulfato ferroso e vacinação antitetânica).

Análises realizadas em nível de município mostram de forma bastante evidente que a ESF teve efeito positivo sobre a redução da mortalidade infantil em diferentes regiões do país 18. Essa redução pode ser atribuída ao aumento da cobertura da assistência pré-natal e à promoção do manejo adequado de doenças comuns na infância, ambos promovidos por essa estratégia ${ }^{6}$. Vale destacar, nessa mesma direção, que a ESF por definição prioriza localidades com maior potencial de impacto, que são os municípios pobres e as áreas pobres de municípios maiores, como é o caso de Rio Grande. Nesse município, em pouco menos de dez anos, a ESF passou a cobrir cerca de $30 \%$ da população, o que representa cerca de metade da população mais pobre do município. Considerando que este estudo incluiu praticamente todas as gestantes que tiveram filho ao longo de um ano no município e que a taxa de fecundidade entre os pobres é maior, é licito pensar que a maior cobertura observada para diversos indicadores da assistência pré-natal alcance níveis até então experimentados somente por usuárias do setor privado.

Esses resultados são interessantes ao SUS, mas carecem de consistência. Há necessidade de realização de estudos semelhantes a este em outras localidades, a fim de verificar se a cobertura aqui observada não foi um achado isolado. Por fim, ao ESF cabe consolidar o nível ótimo de cobertura alcançado para alguns indicadores, aumentar a cobertura para os demais, melhorar a qualidade dos serviços oferecidos e vencer o desafio de reduzir as desigualdades socioeconômicas na mortalidade infantil, ainda bastante evidente em nosso país 6 . 


\section{Resumo}

Este estudo teve por objetivo avaliar a assistência recebida durante o pré-natal nos setores público e privado em Rio Grande, Rio Grande do Sul, Brasil. Aplicou-se questionário padronizado a todas as mães residentes nesse municipio, cujos filhos nasceram nas duas únicas maternidades locais entre 10 de janeiro e 31 de dezembro de 2010. Os locais de consultas avaliados no setor público foram as unidades básicas de saúde (UBS) com e sem Estratégia Saúde da Família (ESF) e os ambulatórios; no setor privado foram as clínicas de convênio e os consultórios particulares. Utilizou-se o teste qui-quadrado para comparar proporções. A taxa de respondentes foi de 97,2\% (2.395 em 2.464). Dentre as 23 variáveis e indicadores avaliados nesses locais, sete mostraram nítida vantagem para mães que consultaram na ESF e seis para mães atendidas em clínica de convênio e consultório particular. Quatro variáveis mostraram cobertura praticamente universal nos cinco locais estudados. A assistência pré-natal mostrou melhor cobertura para gestantes atendidas no setor privado. Gestantes atendidas na ESF apresentaram cobertura semelhante àquela observada no setor privado.

Cuidado Pré-Natal; Avaliação de Serviços de Saúde; Cobertura de Serviços de Saúde

\section{Colaboradores}

J. A. Cesar delineou o estudo, preparou o questionário, supervisionou o trabalho de campo, a entrada e foi responsável pela análise de dados e redação final do artigo. A. T. Sutil auxiliou na preparação e análise dos dados, revisão da bibliografia e redação final do artigo. G. B. Santos colaborou na preparação do banco de dados e redação final do artigo. C. F. Cunha contribuiu na entrada e análise de dados e na redação final do artigo. R. A. Mendoza-Sassi participou da definição do delineamento, análise de dados e redação final do artigo.

\section{Agradecimentos}

À Secretaria Municipal de Saúde de Rio Grande e à direção do Hospital Universitário da Universidade Federal do Rio Grande (HU-FURG) e da Associação de Caridade Santa Casa do Rio Grande (ACSCRG) e ao Conselho Nacional de Desenvolvimento Científico e Tecnológico (Edital Universal CNPq/2009).

\section{Referências}

1. Victora CG, Cesar JA. Saúde materno-infantil no Brasil: padrões de morbidade e possíveis intervenções. In: Rouquayrol MZ, Almeida-Filho NM, organizadores. Epidemiologia e saúde. 6a Ed. Rio de Janeiro: Editora Medsi; 2003. p. 415-67.

2. Barros FC, Victora CG, Matijasevich A, Santos IS, Horta BL, Silveira MF, et al. Preterm births, low birth weight, and intrauterine growth restriction in three birth cohorts in Southern Brazil: 1982, 1993 and 2004. Cad Saúde Pública 2008; 24 Suppl 3:S390-8.
3. Ribeiro ER, Guimarães AM, Bettiol H, Lima DD, Almeida ML, Souza L, et al. Risk factors for inadequate prenatal care use in the metropolitan area of Aracaju, Northeast Brazil. BMC Pregnancy Childbirth 2009; 9:31.

4. Área Técnica da Mulher, Secretaria de Políticas de Saúde, Ministério da Saúde. Programa de Humanização no Pré-natal e Nascimento. Rev Bras Saúde Matern Infant 2002; 2:69-71 
5. Departamento de Atenção Básica, Secretaria de Atenção à Saúde, Ministério da Saúde. Atenção básica e saúde da família. http://dab.saude.gov.br/aten caobasica.php (acessado em 22/Jun/2012).

6. Victora CG, Aquino EML, Leal MC, Monteiro CA, Barros FC, Szwarcwald CL. Maternal and child health in Brazil: progress and challenges. Lancet 2011; 377:1863-76.

7. Nascimento LFC. Perfil de gestantes atendidas nos períodos pré-natal e perinatal: estudo comparativo entre os serviços público e privado em Guaratinguetá, São Paulo. Rev Bras Saúde Matern Infant 2003; 3:187-94.

8. Domingues RMSM, Hartz ZMA, Dias MAB, Leal MC. Avaliação da adequação da assistência prénatal na rede SUS do Município do Rio de Janeiro, Brasil. Cad Saúde Pública 2012; 28:425-37.

9. Chrestani MAD, Santos IS, Cesar JA, Winckler LS, Gonçalves TS, Neumann NA. Assistência à gestação e ao parto: resultados de dois estudos transversais em áreas pobres das regiões Norte e Nordeste do Brasil. Cad Saúde Pública 2008; 24:1609-18.

10. Coutinho T, Monteiro MFG, Sayd JD, Teixeira MTB, Coutinho CM, Coutinho LM. Monitoring the prenatal care process among users of the Unified Health Care System in a city of the Brazilian Southeast. Rev Bras Ginecol Obstet 2010; 32:563-9.

11. Coimbra L, Figueiredo FP, Silva AAM, Barbieri MA, Bettiol H, Caldas AJM, et al. Inadequate utilization of prenatal care in two Brazilian birth cohorts. Braz J Med Biol Res 2007; 40:1195-202.
12. Cesar JA, Mano PS, Carlotto K, Gonzalez-Chica DA, Mendoza-Sassi RA. Público versus privado: avaliando a assistência à gestação e ao parto no extremo sul do Brasil. Rev Bras Saúde Matern Infant 2011; 11:257-63.

13. Silva IS. Cancer epidemiology: principles and methods. Lyon: World Health Organization/International Agency for Research on Cancer; 1999.

14. Takeda S. Avaliação da qualidade do pré-natal: programa para gestantes em unidade de atenção à saúde [Dissertação de Mestrado]. Pelotas: Universidade Federal de Pelotas; 1993.

15. Silveira DS, Santos IS, Dias-da-Costa JS. Atenção pré-natal na rede básica: uma avaliação da estrutura e do processo. Cad Saúde Pública 2001; 17:131-9

16. Cesar JA, Mendoza-Sassi RA. Avaliando a assis tência à gestação e ao parto no Município de Rio Grande, RS: relatório de pesquisa. Rio Grande: Divisão de População \& Saúde, Universidade Federal do Rio Grande; 2010.

17. Cesar JA, Matijasevich A, Santos IS, Barros AJD, Dias-da-Costa JS, Barros FC, et al. The use of maternal and child health services in three population-based cohorts in Southern Brazil, 1982-2004. Cad Saúde Pública 2008; 24 Suppl 3:S427-36.

18. Macinko J, Guanais FC, Souza MFM. Evaluation of the impact of the Family Health Program on infant mortality in Brazil, 1990-2002. J Epidemiol Community Health 2006; 60:13-9.

Recebido em 13/Fev/2012

Versão final reapresentada em 12/Jul/2012

Aprovado em 07/Ago/2012 